\title{
Tactile perception by friction induced vibrations
}

\author{
Ramona Fagiani ${ }^{\mathrm{a}, \mathrm{b}, *}$, Francesco Massi ${ }^{\mathrm{a}}$, Eric Chatelet ${ }^{\mathrm{a}}$, Yves Berthier ${ }^{\mathrm{a}}$, Adnan Akay ${ }^{\mathrm{c}}$ \\ a Université de Lyon, CNRS, INSA-Lyon, LAMCoS UMR5259, F-69621, France \\ ${ }^{\mathrm{b}}$ University of Rome 'La Sapienza', Department of Mechanics and Aeronautics, via Eudossiana 18, 00184 Rome, Italy \\ c Bilkent University Mechanical Engineering Department, TR-06800 Bilkent, Ankara, Turkey
}

\section{A R T I C L E I N F O}

Article history:

Received 17 December 2010

Received in revised form

15 March 2011

Accepted 22 March 2011

Available online 11 May 2011

Keywords:

Tactile perception

Textiles

Roughness

Friction induced vibrations

\begin{abstract}
A B S T R A C T
When a finger moves to scan the surface of an object (haptic sensing), the sliding contact generates vibrations that propagate in the finger skin activating the receptors (mechanoreceptors) located in the skin, allowing the brain to identify objects and perceive information about their properties. The information about the surface of the object is transmitted through vibrations induced by friction between the skin and the object scanned by the fingertip. The mechanoreceptors transduce the stress state into electrical impulses that are conveyed to the brain. A clear understanding of the mechanisms of the tactile sensing is fundamental to numerous applications, like the development of artificial tactile sensors for intelligent prostheses or robotic assistants, and in ergonomics. While the correlation between surface roughness and tactile sensation has already been reported in literature, the vibration spectra induced by the finger-surface scanning and the consequent activation of the mechanoreceptors on the skin have received less attention. In this paper, frequency analysis of signals characterizing surface scanning is carried out to investigate the vibration spectrum measured on the finger and to highlight the changes shown in the vibration spectra as a function of characteristic contact parameters such as scanning speed, roughness and surface texture. An experimental set-up is developed to recover the vibration dynamics by detecting the contact force and the induced vibrations; the bench test has been designed to guarantee reproducibility of measurements at the low amplitude of the vibrations of interest, and to perform measurements without introducing external noise. Two different perception mechanisms, as a function of the roughness wavelength, have been pointed out. The spectrum of vibration obtained by scanning textiles has been investigated.
\end{abstract}

(c) 2011 Elsevier Ltd. All rights reserved.

\section{Introduction}

The sensory perception is obtained through an appropriate combination of the different senses, each specialized in decoding specific information, supplying global information. For example, the sight furnishes information on object's position, but it is primarily the touch sense that suggests information related to materials and surface properties. Specifically, in this paper, the attention is focused on the tactile perception because a clear understanding of the tactile sense is essential for many applications: ergonomics of everyday objects, which affects largely their commercial competitiveness; textile quality; identification of surface imperfections; development of tests for evaluating the tactile sensitivity during diagnosis or monitoring in rehabilitation processes; design of tactile communication devices; development of artificial tactile sensors for intelligent prostheses or robotic assistants; development of human-machine interfaces for the interaction with virtual realities or tele-operation systems, such as

\footnotetext{
* Corresponding author at: Université de Lyon, CNRS, INSA-Lyon, LaMCoS UMR5259, F-69621, France. Tel.: +33472437962.

E-mail address: francesco.massi@insa-lyon.fr (F. Massi).
}

tele-diagnosis or microsurgery; reproduction of real perception (virtual reality); increase of human perception (augmented reality).

In a human hand, there are different types of afferent units, located under the skin, classified on the basis of their properties, in nociceptive units (detecting thermal and mechanical pain), thermoreceptive units (thermal information) and mechanoreceptive units (responding to mechanical excitations) [1,2]. Focusing our attention on the touch sensation, mechanoreceptors, activated by vibrations induced from the finger scanning, are central to tactile perception.

Due to the mechanical loads generated by a contact, the fingertip skin surface is deformed. At any instant of time, during contact, there is a space-time variation of the stress state that causes the mechanoreceptors to respond with an appropriate space-time variation of their discharge rate [3].

Thus, the key to the human tactile sensing is contained in the frictional properties of skin and in the biomechanics of skin and subcutaneous tissues excited by the vibrations induced from the finger/surface scanning [4]. The frictional properties are influenced by hydration, lipid film, surface structure, subject age, anatomic site, race and gender, which characterize the physicochemical and mechanical properties of the skin. Moreover, the human skin has viscoelastic properties that generate a 
dependence of friction behavior on the load, contact area and elastic modulus. Friction is mainly determined by adhesion, implying that the friction coefficient increases with load [5,6].

The activation of mechanoreceptors also depends on the direction of relative sliding motion [7], due both to nonuniform distribution of mechanoreceptors in the skin and to shape of the fingertip surface [8], as well as the different reaction rates (frequency range and stimuli characteristics) [9-15].

The tactile units are made by an afferent fiber and its unmyelinated ending that distinguishes them as Merkel disk, Meissner's corpuscles, Ruffini endings and Pacinian corpuscles. They are located at different depths in the skin, ranging from the epidermis (Meissner's corpuscles) to the subcutaneous fat layer below the dermis (Fig. 1). As indicated in [12,14], these endings are associated with four different tactile afferents populations, respectively: the SA I (slowly adapting type I), the FA I (fast adapting type I, denoted as RA in the older literature), the FA II (fast adapting type II, denoted also as PC) and SA II (slowly adapting type II).

Based on the literature related to microneurography, psychophysical and neurophysiological studies, it is possible to summarize properties and roles of mechanoreceptors in the tactile perception mechanisms [14]:

- The SA I units, ending practically in the glabrous skin, react from about 2 to $16 \mathrm{~Hz}$, responding to skin indentation with an almost linear discharge related to the indentation depth at least of $1.5 \mathrm{~mm} \mathrm{[15].} \mathrm{The} \mathrm{information} \mathrm{they} \mathrm{transmit} \mathrm{to} \mathrm{the}$ brain is a pressure [12] and a spatial neural image of the spatial structure of the object surface. They are sensitive to fine spatial details $(0.5 \mathrm{~mm})$ and are well able to code curvatures [9], edges, corners (because of their linear response to strain energy density) without regard to normal contact force [13] or contact area [16]. Their spatial resolution is minimally influenced by the scanning velocity up to $80 \mathrm{~mm} / \mathrm{s}$ [17].

- The FA I units, located in the skin at a depth of 0.5-0.7 mm, are easily excited between 3 and $40 \mathrm{~Hz}$, and produce the sensation of flutter [11] transmitting a neural image of skin motion to the brain. They are insensitive to static skin deformation and very low frequency vibrations because of their structure. They are most sensitive to transient deformation and low frequency vibration occurring during the increase and decrease of skin indentation [15].

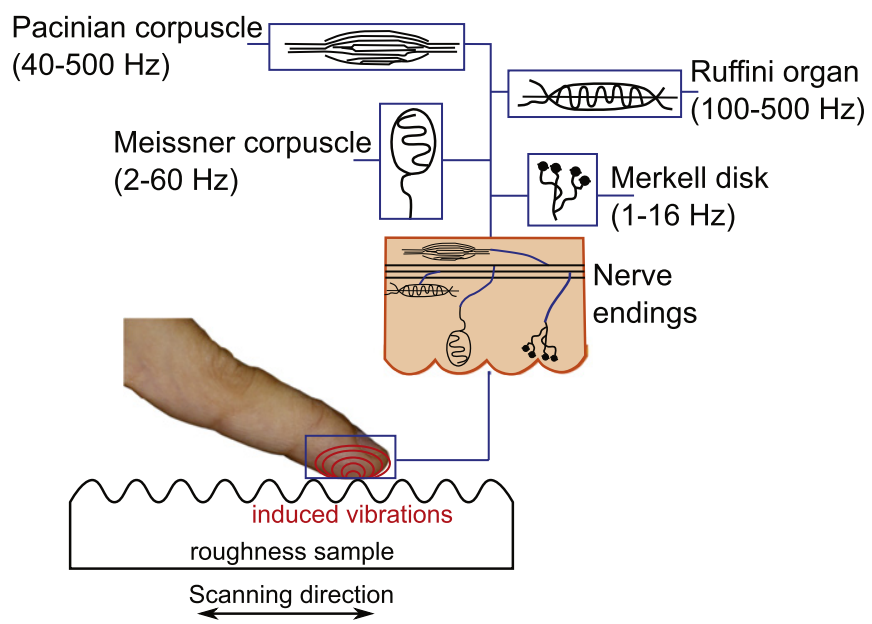

Fig. 1. Schematic representation of the contact between finger and object surface and scheme of the mechanoreceptors position into the skin. The scanning direction is along the line of the finger (from left to right, with respect to the figure).
- The FA II units are the largest in size and are located in the deeper dermis layer, $1.5-2.0 \mathrm{~mm}$. They have great response capabilities, responding to $10 \mathrm{~nm}$ of skin motion at $200 \mathrm{~Hz}$ and strong filtering the low frequency stimuli [18]. For these properties, they are very sensitive to mechanical transients and vibrations of higher frequencies. They react in a range of 40-500 Hz, with the highest sensitivity around $300 \mathrm{~Hz}$ [12].

- The SA II units, characterized by an appreciable sensitivity to lateral stretching of the skin with a pronounced directionality (caused by the fact that their long axis is parallel to the skin surface), react from 100 to $500 \mathrm{~Hz}$, inducing a buzzing sensation [19]. They contribute to the perception of motion direction, hand shape, finger position [20] and contact force [21] through the pattern of skin stretch (i.e. it is possible to generate the finger flexion sensation simply stretching the skin) $[22,23]$.

When there is no relative motion between skin and an object, only slow adapting units are activated in a continuous way. The fast adapting (FA) mechanoreceptors are activated at the beginning of a contact, while the slowly adapting types respond to initial contact and continue to respond throughout the whole contact period. In a static contact, the perception of the surface texture is degraded, i.e. the discrimination of relatively smooth surfaces is practically impossible and the coarse surfaces are perceived as smoother, but the capacity to discriminate changes in material properties, related to their thermal properties, remains [24].

When a relative movement takes place, the slow adapting (SA) units' activation occurs and the fast adapting units are activated by the vibrations induced from the dynamic contact.

Thus, relative motion between a surface and a finger under adequate applied force and scanning speed permits perception of the surface characteristics. The perception mechanism of roughness is based on the "duplex model of tactile roughness perception" [25-35]. These works distinguish between the perception of fine textures (spatial period is smaller than $100 \mu \mathrm{m}$ ), highlighted by vibrations, and the coarse ones (spatial period is bigger than $200 \mu \mathrm{m}$ ), characterized by a "single spatial intensive code", mediated by SA I afferents. This theory has been supported experimentally by several neurophysiological studies. In other words, tactile characterization of fine texture is related to the power of vibrations induced by the dynamic contact with the skin and it is perceived by Pacinian afferents. On the other hand, the coarse textures are transformed into a spatial code generated by the firing variation of the SA I afferents. The results of the induced vibration spectra reported in Section 3 agree with this duplex model of tactile perception and highlight the role of fingerprint roughness on the frequency filtering mechanism. An interesting work investigating the filtering effect of fingerprints is reported in [36].

In this context, it is necessary to perform appropriate experiments to find out the frequency characteristics of the vibrations induced by surface scanning, looking for how tactile sensing is related to the measured frequency spectra. In fact, while the friction aspects of the scanning finger-surface contact are quite well defined (several studies on the effects of loads [6,37], pressure, finger inclination respect the surface, moisturizers [38-41] are reported in the literature), the induced vibration spectra and the consequent activation of the mechanoreceptors on the skin were rarely investigated.

The analysis of vibration spectra induced by scanning the surface can contribute to the definition of objective indexes about the surface perception (softness, textile quality, perceived roughness, etc.). This paper presents a direct approach, investigating the induced vibration spectra. First the design and validation of an 
appropriate bench test, named TriboTouch, is presented. Then, experimental tests characterizing the vibration spectra with respect to scanning speed and surface roughness are presented and the role of fingerprints on the duplex model of tactile perception is highlighted. Finally, the vibration spectra characteristic of textiles is presented and linked to the textile features.

\section{The TriboTouch set-up}

\subsection{Experimental test bench}

The designed experimental set-up (Fig. 2), named TriboTouch, carries a sample surface (a) translated by a compliant mechanism (b), which allows linear motion of the sample without the involvement of any other sliding contact. Specifically, the compliant guide can be approximated by a rigid-body mechanism consisting of rigid links connected to each other by flexible joints (c).

For system stability, two nominally identical compliant mechanisms are placed symmetrically respect to the median plane and slightly tilted toward each other, as shown in Fig. 2. Each element is a double parallelogram that allows for a linear translation of the upper rigid segment where the sample is mounted (a). The flexible joints (c) approximate pin joints between the segments of the double parallelograms and are designed to allow for a linear displacement of the surface sample up to $100 \mathrm{~mm}$. The horizontal translation of the sample is realized by a linear voice coil actuator (d), which, through a feed-back control and a TTL linear encoder, allows imposing the desired scanning velocity. The fingertip is fixed while the scanned surface is moved by the sample translation.

In this paper the behavior of the right-hand index finger has been reported, considering the effects of scanning speed and surface roughness width on the resulting vibration spectra. The difficulty of measuring local dynamics (at the contact region between skin and sample surface) without modifying the contact led to the choice of measuring the global dynamics on the fingertip nail.

Two mono-axial force transducers (e), detecting the normal force, are placed below the sample for measuring the global contact force. A small accelerometer was mounted on the finger nail to measure the finger vibrations induced by the scanning with the test surface (Fig. 3 ). The negligibility of the measurement error introduced by the added mass of the accelerometer was previously verified throughout the comparison between the signal obtained with the accelerometer and the one detected with a laser vibrometer [42].

The study of a finger that moves on a surface involves difficulties that are related to the material characteristics and to the measurements themselves. Because the amplitudes of vibrations generated by the scanning with a finger are very low, it is difficult to isolate them from the parasitic vibrations of the experimental set-up and to detect them without significant alteration.

For this reason it has been decided to employ compliant mechanisms and a linear voice coil actuator. By definition, compliant systems transfer motion, force or energy gaining their mobility from the deflection of flexible members rather than from movable joints. The flexure hinge, a thin member that provides relative motion between two adjacent rigid members through flexing [43], is obtained machining a blank piece of material, thus avoiding the need for any sliding surface and, thus, any friction losses or noise from the joints.

On the other hand, a compliant mechanism is very sensitive to fatigue and overloading. The compliant system and its hinges have been dimensioned to have a linear displacement of the surface sample up to $100 \mathrm{~mm}$. In order to obtain such excursion of the tangential displacement, the flexible joints length depends on

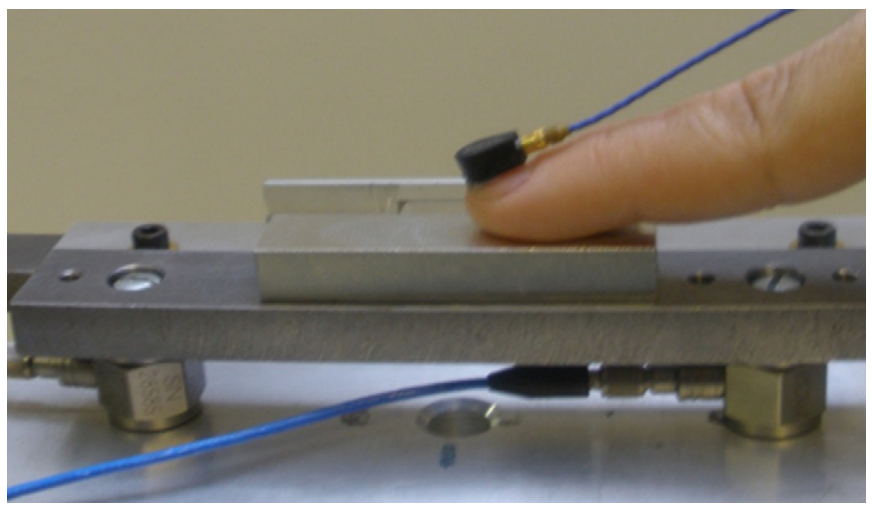

Fig. 3. Experimental set-up configuration used for the measurement.
A

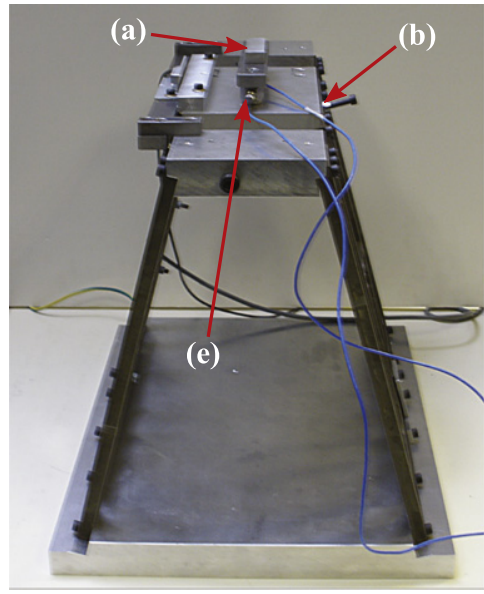

(a) Surface sample;

(b) Compliant mechanisms;

(c) Flexible joint;

B

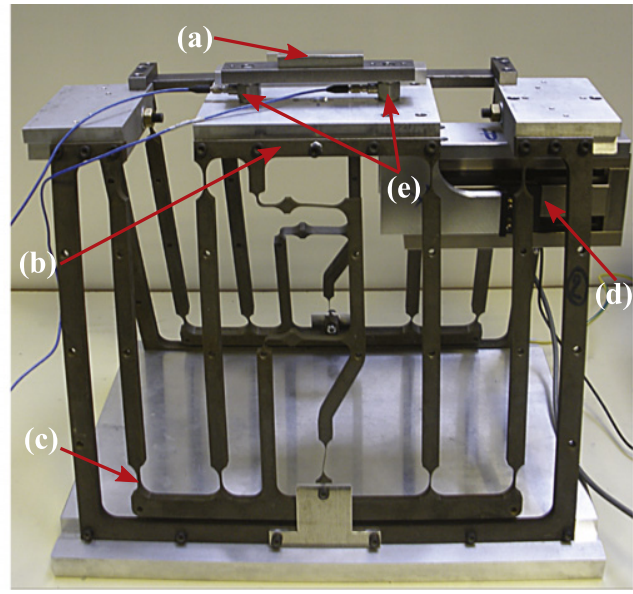

(d) Linear voice coil actuator;

(e) Force transducers;

Fig. 2. Front (A) and side (B) view of the experimental set-up (scale 1:6). 


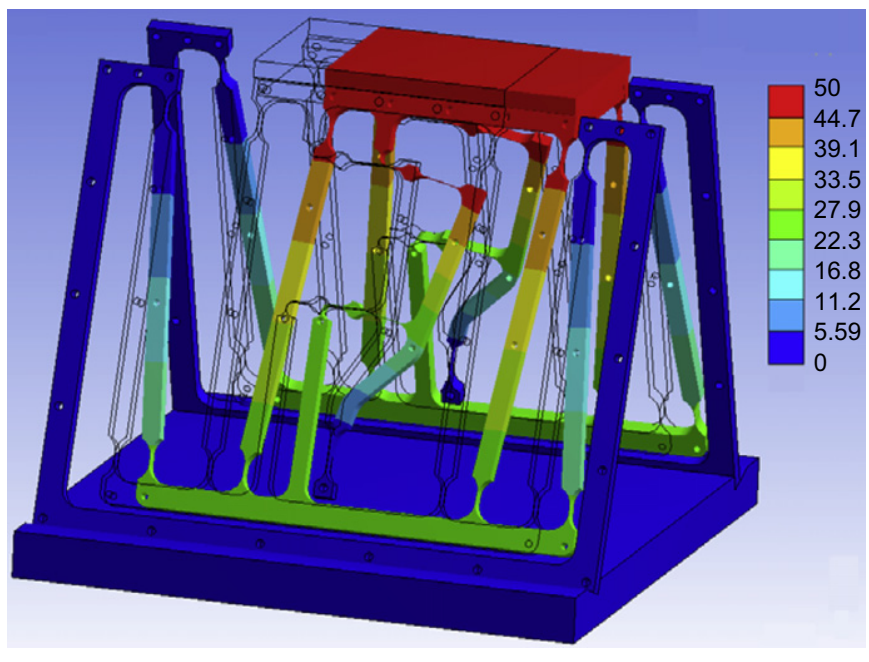

Fig. 4. FEM simulation of the system displacement.

the joint position, 6 or $20 \mathrm{~mm}$. This solution introduces an error in the kinematics of the mechanism, since the flexible joints cannot act as perfect pivots. Nevertheless, the final error calculated and measured on the non-planarity of the linear translation of the surface sample is of about $10 \mu \mathrm{m}$, and is negligible for the tests performed for this study. Fig. 4 shows the compliant mechanism configuration when a tangential force of $2 \mathrm{~N}$ is applied and the maximum displacement is obtained.

A linear voice coil actuator was used in the test bench to provide the desired motion. In this manner, the sample is moved without any direct contact between the dynamic and static part of the actuator, i.e. without sliding vibrations. A high displacement resolution $(0.5 \mu \mathrm{m})$ is obtained using a feed-back position control and a linear encoder. In order to have a better control of the normal force applied by the finger and of the scanning speed, the finger position is fixed and the surface sample is translated at a constant speed using the linear voice coil actuator. It is practically impossible to maintain the normal force perfectly constant during surface scanning.

Only tests with slight variations of the normal force have been retained for the analysis. The applied normal force varies between the subjects in function of the gender, and the age, and generally falls in the range $1.5 \pm 0.7 \mathrm{~N}[6,38]$. During the scanning of the sample the subject, standing close to the experimental set-up, leans his elbow on a rigid planar surface to limit the unintentional movements during the measurements and to have a better force control.

The accelerometer detecting the vibrations is mounted on the finger nail. In order to account for the different points of measurement with respect to the fingertip surface, tests have been performed to obtain the finger transfer function by exciting the fingertip contact surface with a shaker and measuring the response with the accelerometer on the finger nail. The results show high reproducibility for the same subject and similar behavior between different subjects [44]. The calculated spectra are then calculated with the measured transfer function.

\subsection{Test bench validation}

Preliminary tests were out to verify that the proposed set-up is able to detect the desired vibrations. For this purpose, nine types of surface samples with sinusoidal roughness, with $R_{a}$ ranging from 0.64 to $5.2 \mu \mathrm{m}$, and oriented along the transverse direction with respect to the scanned surface, have been used (Table 1 ). The periodical roughness is obtained by milling the surfaces with different milling cutters. Large values of the cutter radii have been chosen to minimize the curvature of the roughness ridges. For a
Table 1

Values of the samples roughness used for the measurements.

\begin{tabular}{llll}
\hline $\begin{array}{l}\text { Roughness } \\
\text { sample }\end{array}$ & $\begin{array}{l}\text { Roughness } \\
\boldsymbol{R}_{\boldsymbol{a}}(\boldsymbol{\mu \mathrm { m } )}\end{array}$ & $\begin{array}{l}\text { Wavelength } \\
(\mathbf{m m})\end{array}$ & $\begin{array}{l}\text { Rapport } \lambda \text { surface/ } \\
\boldsymbol{\lambda} \text { fingertip }\end{array}$ \\
\hline$R_{1}$ & 0.64 & 0.3 & 0.27 \\
$R_{2}$ & 1.0 & 0.15 & 0.33 \\
$R_{3}$ & 1.4 & 0.78 & 0.88 \\
$R_{4}$ & 1.9 & 0.66 & 1.47 \\
$R_{5}$ & 2.5 & 0.87 & 1.84 \\
$R_{6}$ & 3.3 & 1.10 & 2.44 \\
$R_{7}$ & 3.7 & 1.4 & 3.04 \\
$R_{8}$ & 4.5 & 1.71 & 3.80 \\
$R_{9}$ & 5.2 & 2.17 & 4.82 \\
Fingertip & 2.52 & 0.45 & \\
\hline
\end{tabular}

periodic roughness, the vibration frequency can be found as a function of the scanning speed and roughness spatial period (width), ranging from 0.3 to $2.17 \mathrm{~mm}$. The selected roughness range covers surfaces perceived practically smooth up to surfaces with a well-defined roughness. For example, considering everyday objects, $R_{a}=0.64 \mu \mathrm{m}$ is the roughness of an aluminum can and $R_{a}=5.2 \mu \mathrm{m}$ is a type of bottle cork.

Six different scanning velocities have been used, from 10 to $60 \mathrm{~mm} / \mathrm{s}$, representing the range of scanning speed normally employed by people judging a surface [45-48].

Each surface sample was investigated, at each scanning velocity, by different subjects, with the right-hand index finger. All the experiments took place at the same temperature $24{ }^{\circ} \mathrm{C}$ and in the same environmental conditions. The skin was untreated, meaning that no moisturizers were employed, but, to be sure that the contact was always under the same conditions, the finger skin and the surface sample were cleaned up, only with alcohol, before each measurement.

In order to verify the negligible value of the parasitic noise coming from the set-up operation, the measurements of the acceleration with and without scanning between the fingertip and the surface sample are compared. Specifically, in Fig. 5, the black line shows the vibration detected on the finger while it follows the sample without a relative motion between the two surfaces in contact and thus without any friction; the blue line shows the vibrations induced by friction when the finger stays fixed and the sample is moving. The parasitic noise from the set-up (black line) was found to be negligible with respect to the friction induced vibrations (vibrations of interest) measured when scanning the surface (blue line). The first acceleration peak at $1.1 \mathrm{~s}$ is due to the start of the horizontal displacement of the sample.

As mentioned above, the object texture is perceived by scanning it with a fingertip because this information is contained in the vibrations induced by the dynamical contact between the finger and the surface. The induced vibrations depend primarily on the roughness of the object surface and on the fingertip, their material properties, the speed at which the fingertip moves on the surface and the movement direction.

The measurements performed on samples with periodical roughness show well-defined frequency peaks that depend on the period of the roughness on the sample.

Fig. 6 shows the fast Fourier transforms (FFTs) of the spectra of the acceleration measured on the finger for six different scanning speeds on the same roughness sample $\left(R_{2}\right)$ divided by the finger transfer function. Increasing the scanning speed from 10 to $60 \mathrm{~mm} / \mathrm{s}$ the peaks on the frequency spectra cover larger values and their amplitude rises. The same behavior can be found for all the surface samples and for different subjects, confirming that the designed experimental set-up is suitable for measuring and investigating the induced vibrations. 


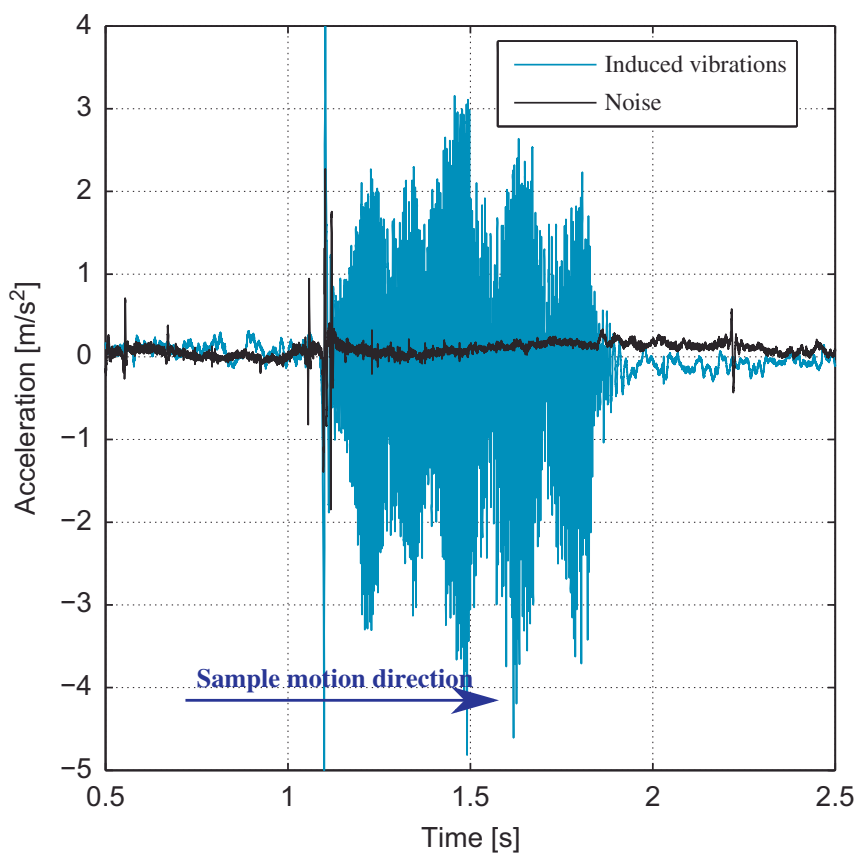

Fig. 5. An example of the comparison between the acquired acceleration signal of the vibrations induced by the fingertip/surface scanning and the parasite noise from the set-up functioning. (For interpretation of the references to color in this figure, the reader is referred to the web version of this article.)

\subsection{Friction coefficient}

The designed apparatus, developed from the system presented in [49], allows for calculating the global friction coefficient between fingertip and surface, by monitoring the normal forces at the connection between surface sample and support, and the position of the fingertip. With respect to Figs. 7 and 3, the global contact force between the fingertip and the sample surface can be decomposed in the normal component, $N$, along the direction normal to the sample surface and the friction one, $T$, which acts along the direction tangential to the sample surface, opposite to the motion direction. The global friction coefficient $\mu$ is defined as the ratio between $T$ and $N$.

The surface sample is fixed at the two force transducers that connect it to the linear guide (Fig. 2). If the finger motion lies in the same vertical plain of the two points of connection the problem can be reduced to a planar one and, at each point, three reaction components have to be accounted for: $F_{1}$ and $F_{2}$, which are the force components measured with the force transducers; $R_{1}$ and $R_{2}$, along the tangential direction; and the two moments $M_{1}$ and $M_{2}$ along the $z$ axis (Fig. 7(a)).

Considering the structure as a clamped beam, it is possible to obtain the equilibrium equations:

$T=R_{1}+R_{2}$

representing the force equilibrium along the $x$ axis;

$N=F_{1}+F_{2}$

representing the force equilibrium along the $y$ axis;

$-N(L-d)+\mu N h+F_{2} L-M_{2}+M_{1}=0$
Only the last two equations are useful for this application, and in particular the second one furnishes the desired friction coefficient:

$\mu=\frac{M_{2}-M_{1}-F_{2} L+\left(F_{1}+F_{2}\right)(L-d)}{\left(F_{1}+F_{2}\right) h}$

While $F_{1}, F_{2}, L$ and $h$ are directly measured during the scanning of the fingertip on the sample surface, $M_{1}$ and $M_{2}$ are unknown. They can be calculated as a function of the normal force $N$, the dimensions of the structure and the finger position, considering the hyperstatic problem shown in Fig. 8, where the friction force $T$ gives the moment $M_{T}$ at the line between the two points of connection with the frame:

$M_{T}=T h=\mu N h$

The displacement of the sample is driven by a trapezoidal function of the actuator speed. The parameter $d$ contains the information about the fingertip position, which is calculated as

$d=p_{0}+v t$

where $p_{0}$ is the initial distance of the fingertip from the force transducer when the sample reaches the constant value of the actuator speed, $v$ is the actuator speed and $t$ is the time. The position can be as well monitored experimentally by the position of the linear encoder.

The differential equations that yield the shear $(V)$ and the bending moment $(M)$ as a function of the slope $\left(y^{\prime}\right)$, the deflection $(y)$, the Young modulus $(E)$ and the inertia moment $(I)$,

$V=\frac{d M}{d x}, \quad M=E I \frac{d y^{\prime}}{d x} \approx E I \frac{d(d y / d x)}{d x}=E I \frac{d^{2} y}{d x^{2}}$

can be used to determine the shear and bending moment diagrams and the equations of the elastic curve for any beam element subject to a given set of loading. Through successive integration, these equations are used to determine the slope and deflection function of a segment of beam. The integrations constants can be determined enforcing boundary and/or continuity conditions of the beam segment. Boundary conditions are imposed at point of support for the structure and continuity conditions at point common to two adjacent beam segments.

Specifically, in this case (Fig. 8), there are four beam elements to consider: $A B, B C^{\prime}, C^{\prime} C$ and $C D$. The following boundary and continuity conditions are assumed:

$$
\begin{array}{lllll}
\text { Boundary conditions: } & \multicolumn{3}{c}{\text { Continuity conditions: }} \\
y_{A B}^{\prime}(A)=0 & y_{C D}^{\prime}(D)=0 & y_{A B}^{\prime}(B)=y_{B C^{\prime}}^{\prime}(B) & y_{B C^{\prime}}^{\prime}\left(C^{\prime}\right)=y_{C^{\prime} C}^{\prime}\left(C^{\prime}\right) & y_{C^{\prime} C}^{\prime}(C)=y_{C D}^{\prime}(C) \\
y_{A B}(A)=0 & y_{C D}(D)=0 & y_{A B}(B)=y_{B C^{\prime}}(B) & y_{B C^{\prime}}\left(C^{\prime}\right)=y_{C^{\prime} C}\left(C^{\prime}\right) & y_{C^{\prime} C}(C)=y_{C D}(C)
\end{array}
$$

Integrating the equations on each beam element and introducing the boundary and continuity conditions, it is possible to obtain the equations of $M_{1}$ and $F_{1}$ in function of $N, \mu$ and geometric components:

$$
\begin{aligned}
M_{1}= & \frac{N}{\left.(L+2 b(-1+\alpha))(-2 b-L)^{2}+2 b\left(4 b^{2}-6 b L+3 L^{2}\right) \alpha\right)} \\
& \times\left[\left(4 b^{5}(-1+\alpha)^{2}-2 b^{4}(-1+\alpha)^{2}(4 d+L+4 H \mu)\right.\right. \\
& +2 b L(-1+\alpha)\left(-d^{3}+d L^{2}-3 d^{2} H \mu+H L^{2} \mu\right) \\
& +d L^{2}\left(-d^{2}+2 H L \mu+d(L-3 H \mu)\right)-b^{2} L(-1+\alpha)\left(-6 d^{2}+3 d(L-4 H \mu)\right. \\
& +L(L+3 H \mu))+2 b^{3}(-1+\alpha)\left(-2 d^{2}+3 d L(-1+\alpha)\right. \\
& -4 d L H \mu+L(L+3 H(-1+\alpha) \mu))]
\end{aligned}
$$

representing the moment equilibrium at point $A$.

$$
F_{1}=\frac{\left(N\left(4 b^{3}(-1+\alpha)+6 b L(-1+\alpha)(d+H \mu)-3 b^{2}(-1+\alpha)(2 d+L+2 H \mu)+d\left(-2 d^{2}+6 H L \mu+3 d(L-2 H \mu)\right)\right)\right)}{\left((L+2 b(-1+\alpha))\left((-2 b+L)^{3}+2 b\left(4 b^{2}-6 b L+3 L^{2}\right) \alpha\right)\right)}
$$




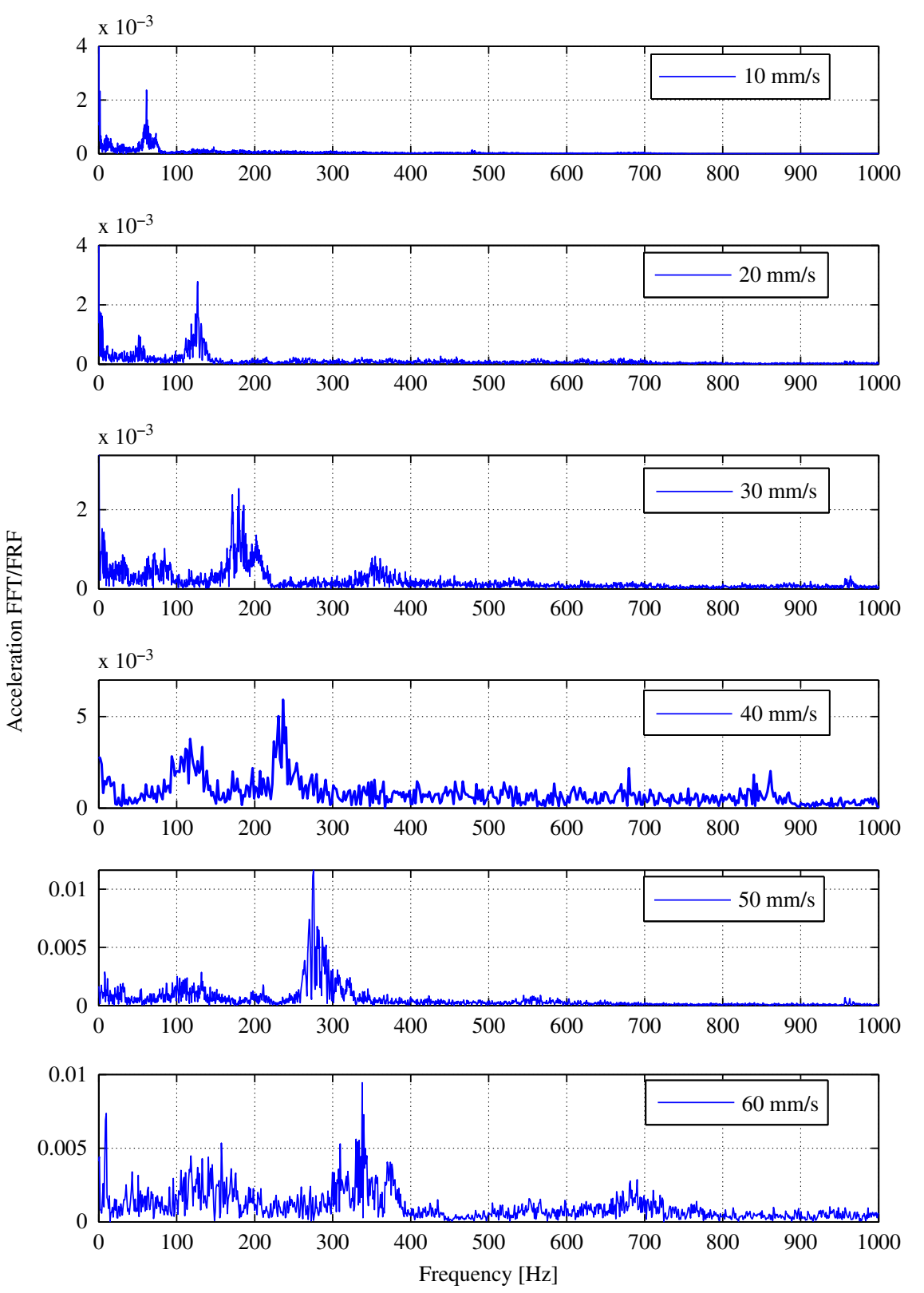

Fig. 6. Acceleration FFTs of the signals obtained from the accelerometer, at different speeds and for $R_{2}$ roughness sample, divided by the finger transfer function.

Then, $M_{2}$ can be calculated from the equilibrium equations and, finally, the friction coefficient can be calculated as a function of the measured forces $F_{1}$ and $F_{2}$ : maintained constant and the sample moves to make the fingertip scan the surface. In this phase the contact moves from one transducer position to the other, so that $F_{1}$ decreases as the

$\mu=\frac{\left.\left(F_{2} L^{3}-\left(\left(p_{0}+v t\right)-L\right)^{2}\left(2\left(p_{0}+v t\right)+L\right) N+4 b^{3}\left(2 F_{2}-N\right)(\alpha-1)+6 b L\left(F_{2} L+\left(\left(p_{0}+v t\right)-L\right) N\right)(\alpha-1)-3 b^{2}\left(4 F_{2} L+2\left(p_{0}+v t\right) N-3 L N\right)(\alpha-1)\right)\right)}{\left(6 h N\left(\left(p_{0}+v t\right)\left(\left(p_{0}+v t\right)-L\right)+b^{2}(\alpha-1)+b(L-L \alpha)\right)\right)}$

where $\alpha$ is the ratio between the inertia momentum $I_{o}$, of the $A B$ element beam cross-section, and $I_{1}$, of the $B C$ element beam crosssection, as shown in Fig. 8(b).

Fig. 9(a) shows an example of the force components detected and the calculated friction coefficient. During the first phase of the test (Phase I) there is no contact between fingertip and sample. At time $t=2.6 \mathrm{~s}$ the fingertip is put in contact with the surface sample (Phase II). During Phase III the normal force is motion takes place and the opposite happens for $F_{2}$; their sum, $N$, remains constant during the measure.

Fig. 9(b) shows the friction coefficient calculated as reported before during the surface scanning of the fingertip skin on a steel sample (Phase III). The skin behavior causes a low decrease of the curve. In fact, during the scanning phenomena like natural skin hydration and the production of cutaneous lipid film take place reducing the friction coefficient $[37,40]$. 
Fig. 10 shows the dependence of the friction coefficient with respect to the scanning speed and the contact load. Fig. 10(a) shows the friction coefficient mean values, averaged on the scanning time, as a function of the scanning speed. As expected [41], the friction coefficient decreases with the increase of the scanning speed. Fig. 10(b) is an example of the friction coefficient behavior with respect to the normal load. The decrease of the friction coefficient with the increasing of normal load suggests the effect of the adhesion mechanism.

Neglecting the moments M1 and M2 (iso-static problem) leads to a relative error up to $30 \%$ in the calculation of the friction
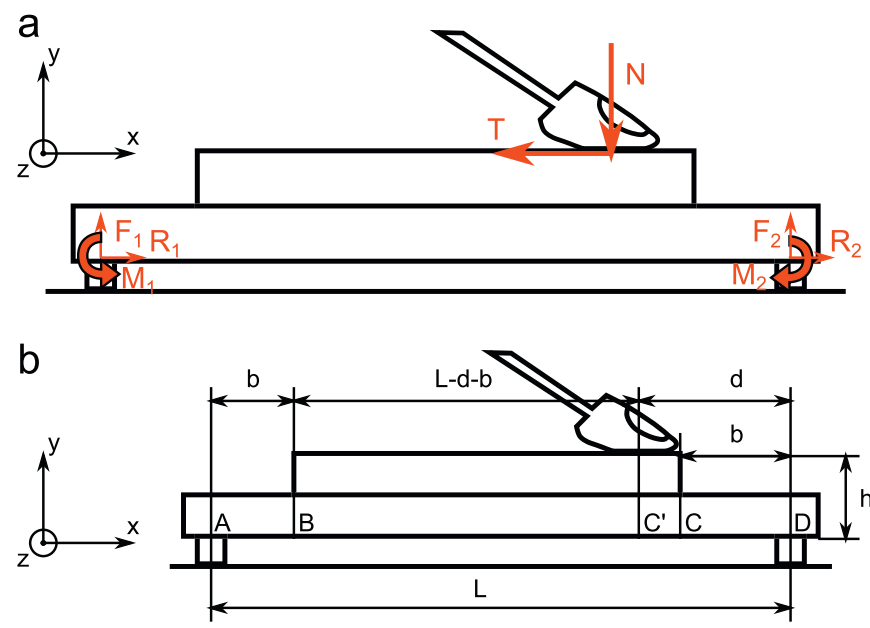

Fig. 7. (a) Scheme of the mechanical actions for the friction coefficient calculus and (b) geometric parameters. coefficient. In order to verify all the hypotheses, measurements of the friction coefficient have been performed with a tri-axial force transducer along a smaller displacement (about $30 \mathrm{~mm}$ for
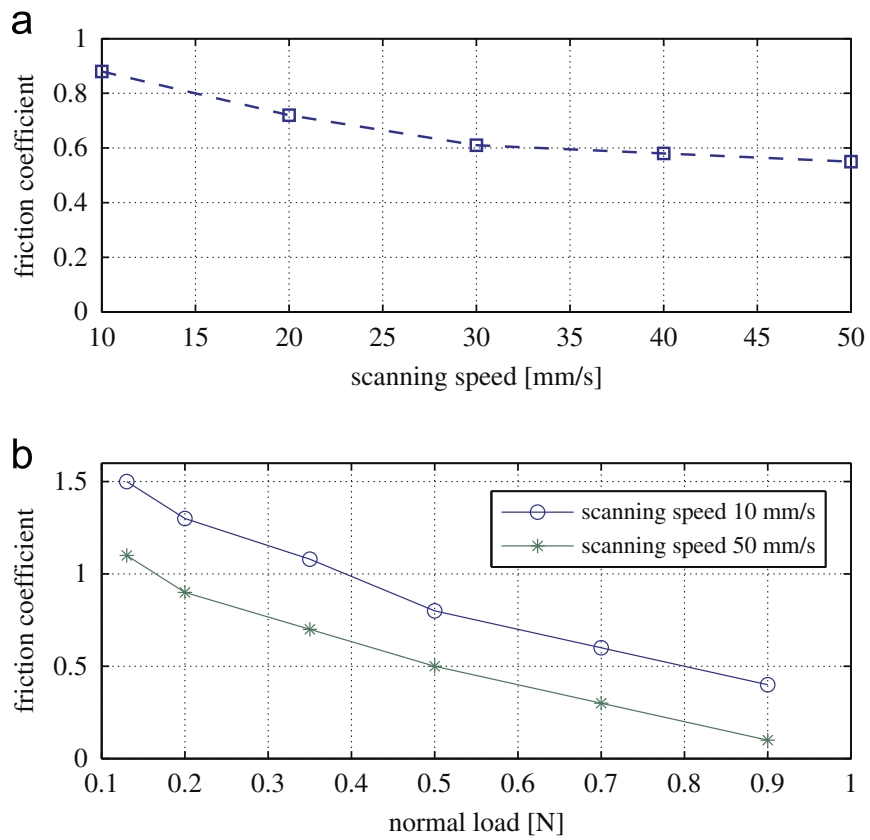

Fig. 10. (a) Friction coefficient between the fingertip and a steel surface sample with respect to the scanning velocity. (b) Friction coefficient between the fingertip and a steel surface sample with respect to the normal load for the extremes scanning speed employed during the measurements.
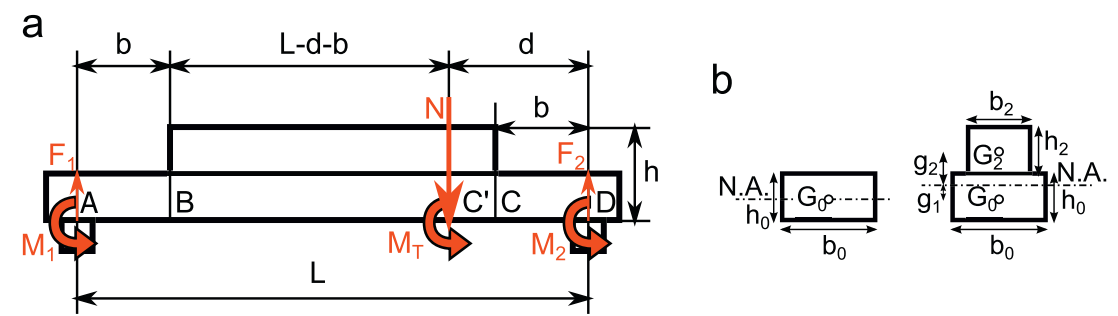

Fig. 8. (a) Hyperstatic problem for the calculus of the flexion component as a function of known parameters and (b) cross-sections of the different beam elements.
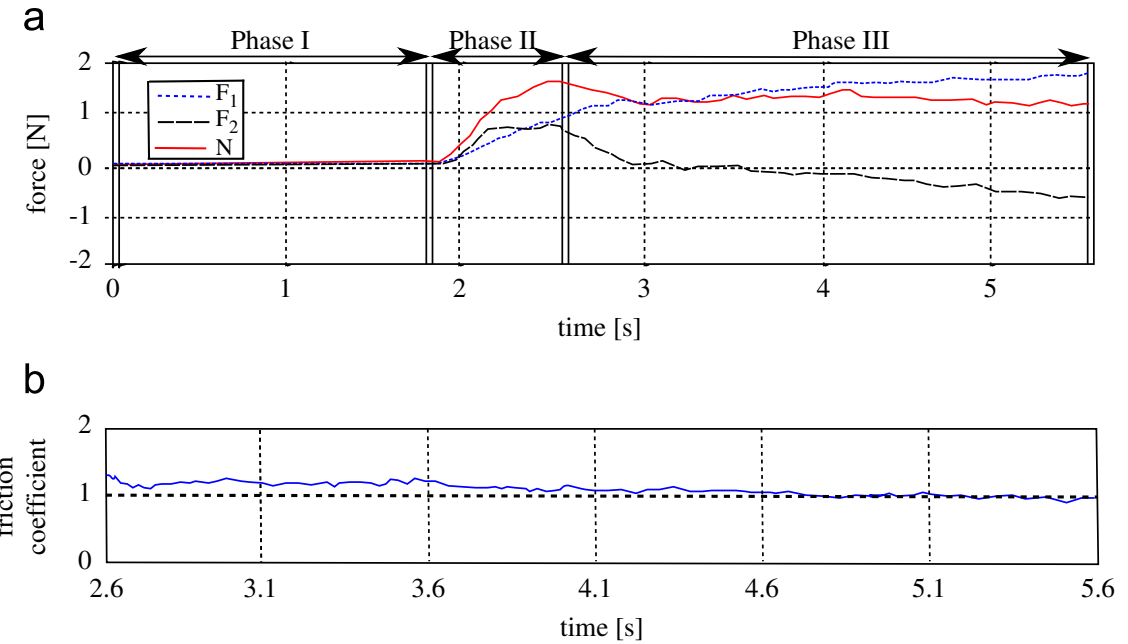

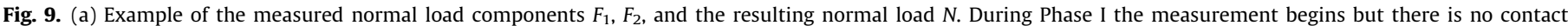

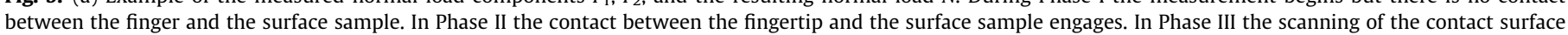

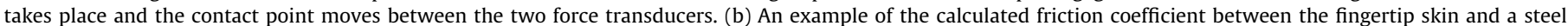
sample during Phase III. 
avoiding the torque on the transducer). The relative error with respect to the measurements obtained accounting for $M 1$ and $M 2$ was found to be less than $4 \%$.

\section{Effect of scanning speed and roughness wavelength}

When dealing with samples that have periodic surface roughness, a well-defined peak in its vibration spectrum is measured, as shown in Fig. 6 [42,44]. The vibration frequency is a function of the scanning speed and of the relationship between the roughness spatial period (width) of the surface samples and of the fingerprint.

Fig. 11 shows the frequency peaks of the induced vibrations (detected by the accelerometer mounted on the finger nail) as a function of scanning speed (for each roughness width), for two different subjects: a female 27 years old (fingerprint wavelength $0.45 \mathrm{~mm}$ ) and a male 32 years old (fingerprint wavelength $0.67 \mathrm{~mm}$ ). The results are reproducible and it can be asserted that the frequency peak value increases linearly with the increase of the scanning velocity.

Fig. 12 shows the measured vibration frequencies for subject no. 1 (fingerprint wavelength $0.45 \mathrm{~mm}$ ) when scanning the surface samples with roughness wavelength equal to $1.4 \mathrm{~mm}$ (stars) and $0.3 \mathrm{~mm}$ (circles). The continuous line, representing the frequencies, is calculated as a ratio of the scanning speed and the roughness spatial periods. When the wavelength of the surface roughness $(0.3 \mathrm{~mm})$ is much smaller than the fingerprint

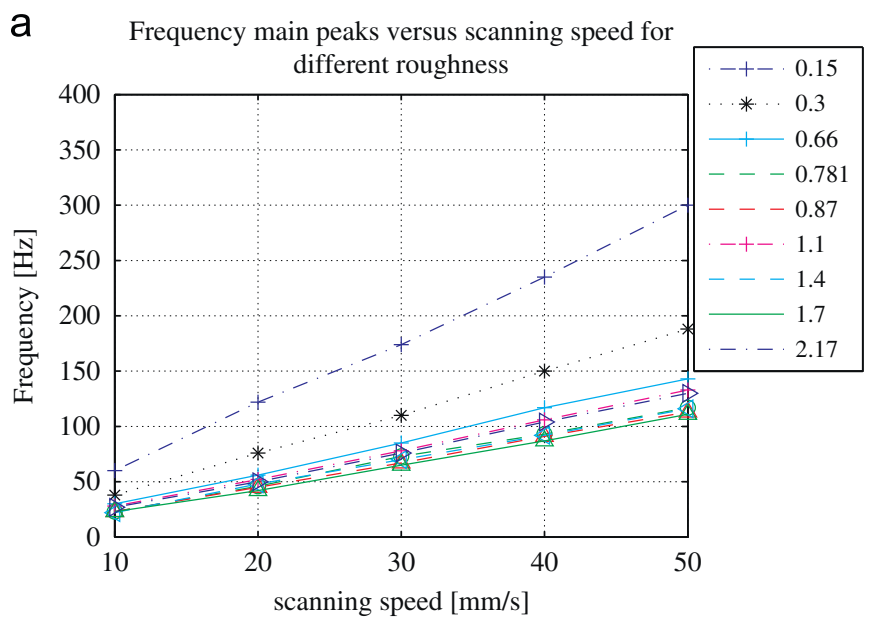

b Frequency main peaks versus scanning speed for

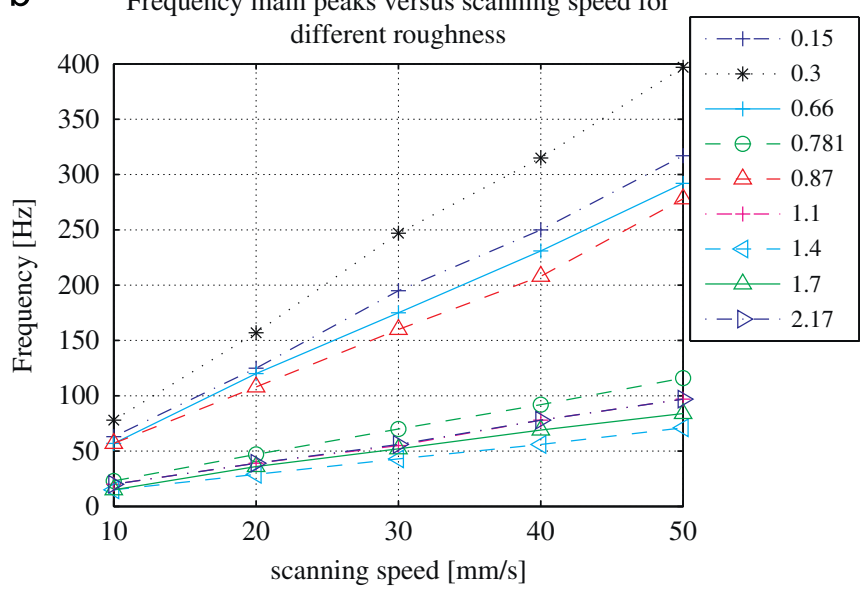

Fig. 11. Relationship between the frequency main peaks and the scanning speed for different roughness samples: (a) subject 1 and (b) subject 2 .

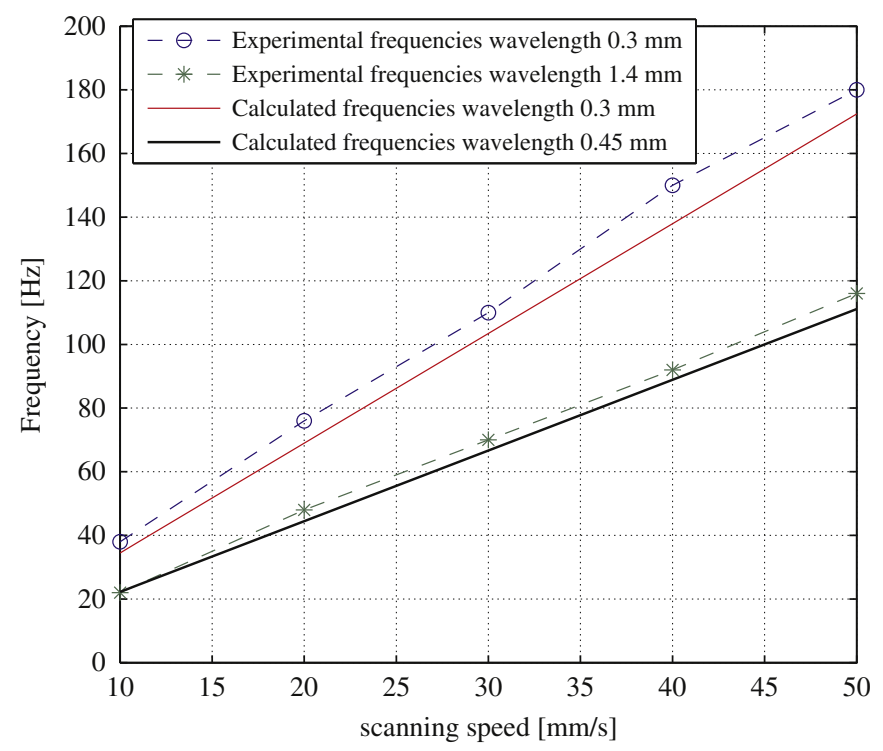

Fig. 12. Comparison between experimental and calculated frequencies.
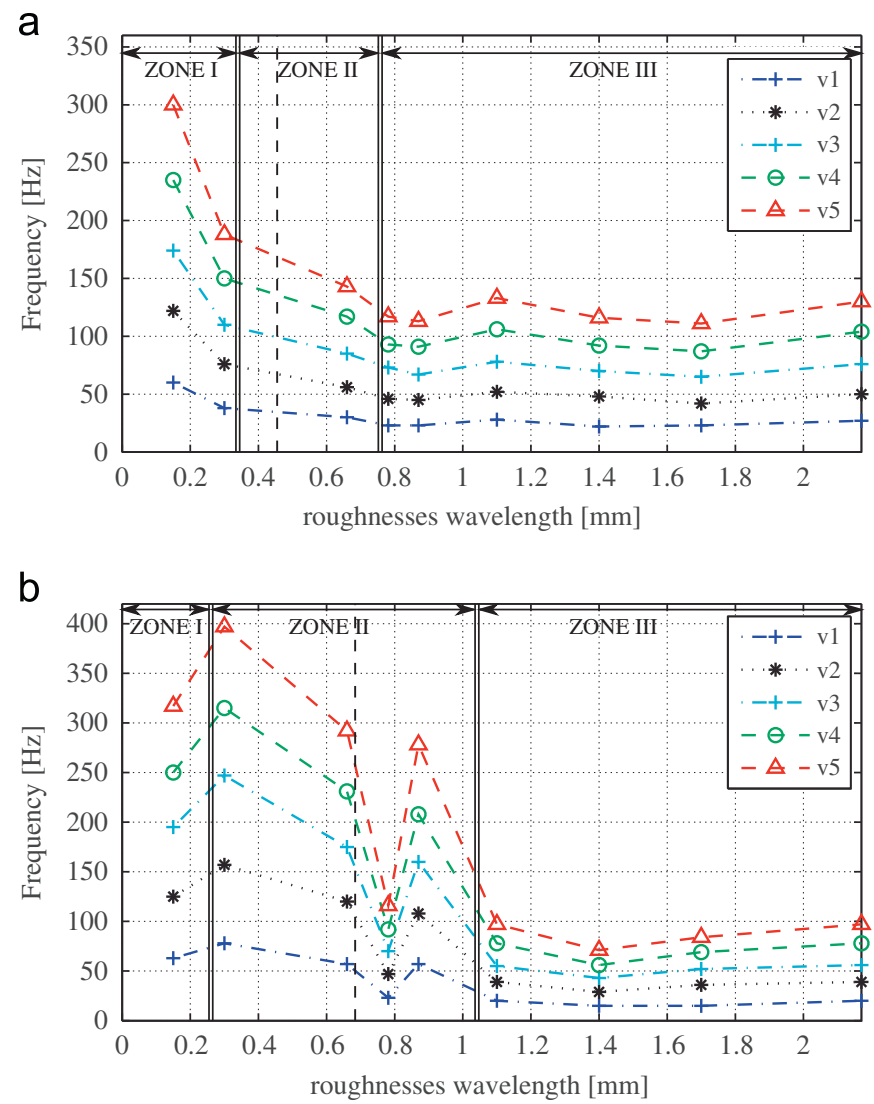

Fig. 13. Relationship between the frequency main peaks and the roughness width for different roughness samples: (a) subject 1 and (b) subject 2 .

wavelength $(0.45 \mathrm{~mm})$, the measured frequencies correspond to the frequencies calculated with surface roughness wavelength. On the contrary, when the surface roughness $(1.4 \mathrm{~mm})$ is much larger than the fingerprint wavelength $(0.45 \mathrm{~mm})$, the measured frequencies correspond to the analytical frequencies calculated with the fingerprint wavelength, i.e. the frequency of the scanning induced vibrations is always function of the smaller wavelength.

A more detailed analysis can be made by examining Fig. 13, which displays the frequency of the induced vibrations as a 
function of sample roughness width (at each scanning speed and for the two subjects).

There is a non-linear dependence of the frequency peak with respect to the roughness width of the sample, resulting from the presence of the fingertip roughness. The final excitation frequency is a function of the mutual roughness, meaning the combination of the fingertip and surface sample roughness. It is possible to distinguish three different zones in function of the fingerprint wavelength:

- ZONE I: The sample surface wavelength is smaller than that of the fingerprint, and the frequency peak value depends primarily on the roughness wavelength of the sample. The same frequencies of the scanning induced vibrations have been measured for the two subjects at each scanning speed.

- ZONE II: The sample surface wavelength is comparable with that of the fingerprint, and the frequency peak is a function of the ratio between the wavelengths of the sample and the fingertip roughness. This is a transition zone of the excitation mechanism provided by the sliding between two quasi-sinusoidal surfaces (the fingertip and the sample). Samples having wavelength close to a multiple of the fingertip roughness semi-period allow for larger value of the excitation frequency, while the others follow the trend shown in ZONE III where the fingertip roughness determines the frequency peak. This different behavior can be associated to the frequency of the impacts between ridges of the fingerprints and the roughness of the sample surface, and thus to the degree of conformability between the roughness of the two sliding surfaces.

- ZONE III: The surface sample wavelength is larger than that of the fingerprint, and the frequency peak value depends primarily on the roughness wavelength of the fingerprints. In ZONE III the measured frequencies are for the same subject, almost constant for all the sample roughness.

The same behaviors have been observed for different subjects; nevertheless, even if the global trend of the spectra as a function of roughness and scanning velocity is the same, the values of the peak frequencies and the slope of the curves shown in Figs. 11 and 13 change because of the differences in fingertip characteristics, as shown in Figs. 11(b) and 13(b). The presented results support the existence of two different mechanisms of perception of surface roughness as a function of the roughness wavelength. While in ZONE I and ZONE II the spectra of the scanning induced vibrations are affected by the surface roughness, in ZONE III the spectra of the induced vibrations do not give information about the surface, because they are only functions of the fingerprint width. During tests with samples in ZONE III both subjects perceived the roughness of the sample as a spatial distribution of the contact pressure between the fingertip and the sample surface, but they could not perceive any differences in the local vibrations. On the contrary, when scanning surface samples of ZONE I and ZONE II neither subjects could perceive any information about the spatial distribution of pressure, but they perceived the different roughness of the samples by the sensation of local vibrations at the fingertip/sample interface.

These results agree with the "duplex model of tactile perception" highlighted by psychophysical and neurophysiologic studies [25-35], and described above.

Recent psychophysics tests, presented by Martinot and dealing with fine texture perception [50], showed an increase of roughness perception when the roughness wavelength is close to the fingerprint's one. He suggested that the "roughness perception depended on the commensurability and conformance between the surface details of both skin and haptic texture at contact". The results presented in this paper confirm the importance of the roughness conformance. Anyway it is still not clear if the different roughness perception comes from frequency cues alone, or from spatial cues due to the localized shear forces. It is the opinion of the authors that both the spatial and the temporal cues affect the perception of the fine textures.

\section{Application to textiles}

The frequency peaks of the measured vibration spectra obtained from periodical roughness samples, comparable with daily object roughness, stay in the frequency range expected from the literature dealing with the perception range of the mechanoreceptors [2-500 Hz].

To generalize this result, tests have been performed with surface samples having isotropic roughness, i.e. without any preferential direction. The isotropically rough surfaces are obtained by sandblasting rectified surfaces with different sandblasting distances, pressures and different grain dimensions. Measurements of induced vibrations resulting from scanning between fingertip and an isotropically rough surface also show that the induced vibration spectra stay in the same frequency range (Fig. 14). In this case, the vibration spectra do not show a mean frequency peak, as with a periodic roughness, because of the non-periodicity of the employed surfaces. Nevertheless, as for the surfaces with periodic roughness, it is possible to observe from the obtained spectra that with increasing the scanning velocity both the frequencies and the amplitude of the induced vibrations increase.

Fig. 15 shows the vibration spectra obtained when the fingertip scans a textile for two different values of the scanning speed.

The vibration spectra obtained when scanning textiles show a frequency behavior, which is a combination of the spectra described previously. In fact, as shown in Fig. 15, there is both a well-defined frequency peak, characteristic of periodic surface

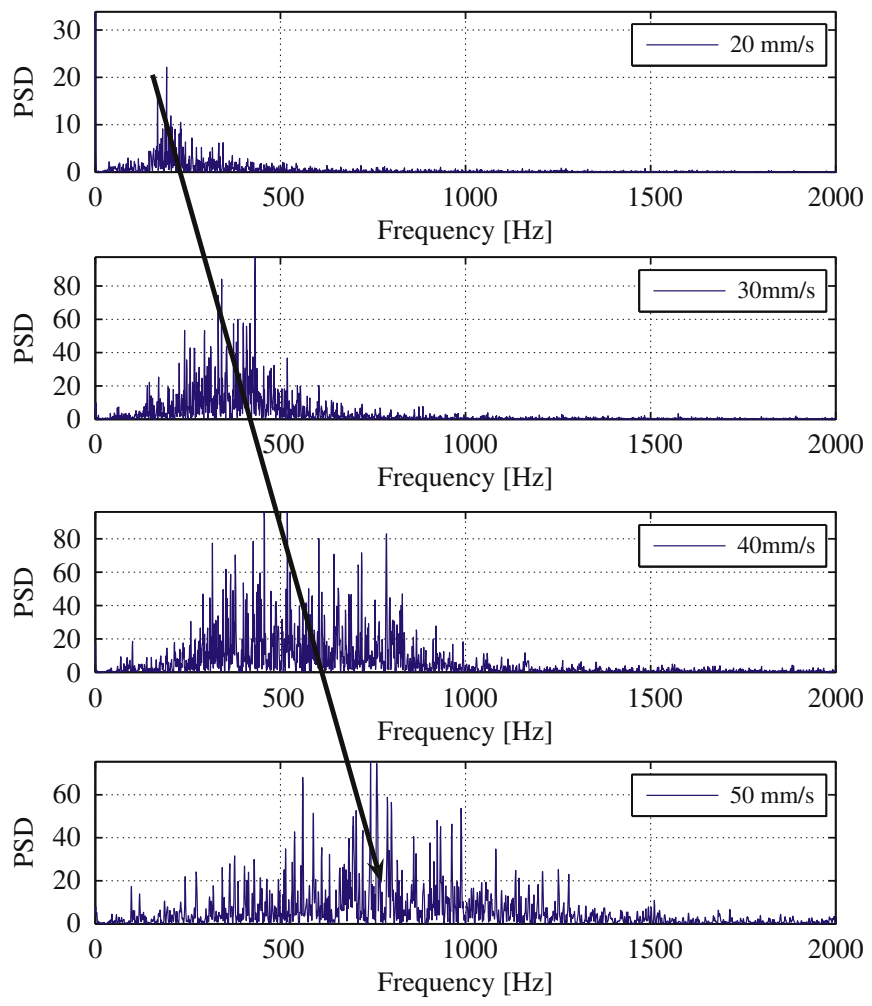

Fig. 14. An example of the acceleration PSD magnitude for the signals obtained from the accelerometer employing an isotropic surface sample. 


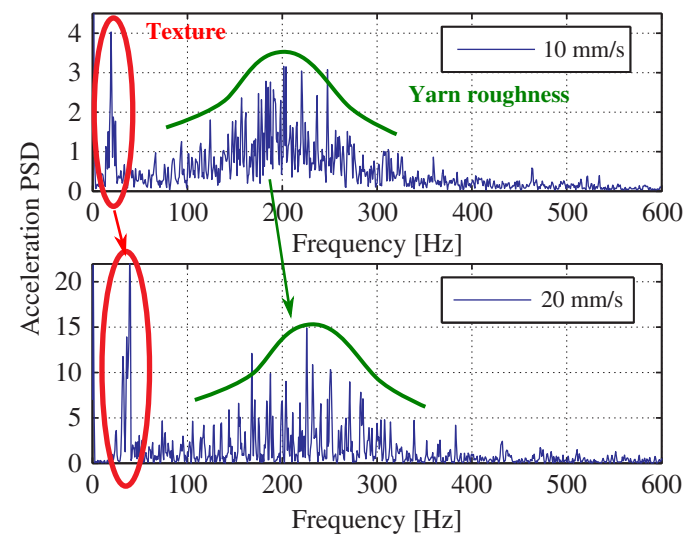

Fig. 15. An example of the acceleration PSD magnitude for the signals obtained from the accelerometer employing a textile sample.

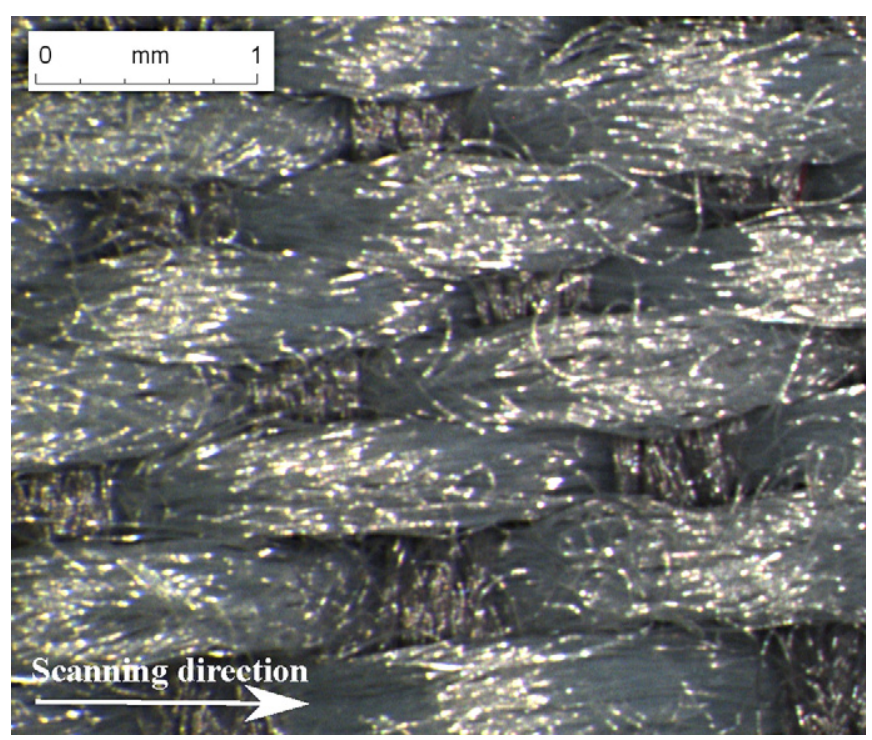

Fig. 16. Woven fabric texture. The texture period is about $2 \mathrm{~mm}$.

roughness, and a larger frequency distribution, characteristic of isotropic roughness. This behavior is due to the structure of the textile (Fig. 16): the well-defined frequency peak is due to the periodicity of the texture of the fabric, while the higher frequency distribution is related to the roughness of the threads composing the texture. For the woven fabric shown in Fig. 16, the texture width periodicity is about $2 \mathrm{~mm}$, which is greater than the fingerprint width; the frequency peak of the induced vibrations corresponds to calculated value for the fingerprint width (see Section 3). This particular feature of the induced vibration spectra resulting from scanning a textile opens a new interest domain of investigation to link the two components of the spectra with the quality perception when touching a textile.

As for surfaces with periodic and random roughness, it is possible to observe that, increasing the scanning velocity, the amplitude of the vibration increases and the induced vibrations move to higher frequencies.

\section{Conclusions}

The mechanoreceptors convert vibrations induced by scanning a surface into electric impulses that are then sent to the brain. Therefore, the perceived surface and material characteristics depend on the contact properties and conditions like material properties, the mutual roughness, the hydration, the scanning speed, etc.

To understand the relationship between surface characteristics and tactile sensation, it is necessary to better understand how the vibrations develop at the interface of the skin and develop a method to measure and analyze them.

This paper describes a unique test bench, the TriboTouch, which has been developed to reproduce the scanning action between finger and surface under controlled parameters, avoiding any other contact surface in sliding, i.e. preventing parasitic vibrations that would affect the measurements.

Using this new apparatus, the influences of scanning speed and roughness wavelength on the vibration spectra have been investigated:

- For all the roughness samples, the acceleration peaks fall between 2 and $500 \mathrm{~Hz}$, which correspond to the sensitivity range of the mechanoreceptors based on physiological data.

- For each contact surface, an increase in speed corresponds both to the shift of the acceleration spectrum toward higher frequencies and to larger magnitudes; for samples with periodic roughness, the frequency peak value increases linearly with an increase in the scanning velocity.

- The dependence of the frequency peak of the induced vibrations with respect to the roughness width of the sample shows three different behaviors related to the ratio between the wavelengths of the sample and the fingerprint:

○ When the sample roughness wavelength is much larger than that of the fingerprint, the spectrum is a function of the fingerprint roughness;

$\bigcirc$ when the sample roughness wavelength is comparable to than that of the fingerprint, the spectrum is a function of the ratio between them;

- When the sample roughness wavelength is much smaller than the wavelength of the fingerprint, the spectrum is a function of surface roughness.

Consequently, two different perception mechanisms are highlighted as a function of the roughness wavelength and vibration spectra: when the wavelength of the surface roughness is smaller or comparable to that of the fingerprint one, the surface roughness is perceived as a result of the vibrations induced by the finger scanning. When roughness wavelength is much larger than that of the fingerprint one, it is perceived as a quasi-static pressure distribution on the fingertip surface. These results agree with psychophysical and neurophysiologic analyses reported in literature.

A preliminary analysis of the spectra of vibrations induced by scanning textiles was also performed:

- Isotropic roughness samples show a vibration spectra characterized by a larger frequency distribution than the periodic one.

- The spectra of the friction induced vibrations obtained when scanning a textile sample show simultaneously:

$\bigcirc$ a vibration frequency peak, characteristic of the periodically rough surfaces, which is due to the texture of the fabric;

$\bigcirc$ a wider frequency distribution, characteristic of isotropic roughness, which is due to the roughness of the textile fibers.

A further investigation will be helpful to understand the role of these two different components of vibration spectra on the quality perception of a textile when 'testing' it with fingertips. 
Future work will be aimed to characterize parameters (indexes) that allow for describing objectively the relationship between the perception of the touched surface and the spectra of the induced vibrations.

\section{References}

[1] Johansson RS, 1978, Tactile sensibility in man. A quantitative study of the population of mechanoreceptive units in the glabrous skin area of the hand, Umea University Medical dissertation, New series no. 35.

[2] Spray DC. Cutaneous temperature receptors. Annual Reviews of Physiology 1986;48:625-38.

[3] Jones LA, Lederman SJ. Human hand function.Oxford University Press; 2006.

4] Akay A. Acoustic of friction. Journal of Acoustical Society of America 2002;111(4):1525-48.

[5] Koudine AA, Barquins M, Anthoine PH, Aubert L, Leveque J-L. Friction properties of skin: proposal of a new approach. International Journal of Cosmetic Science 2000;22:11-20.

[6] Tang W, Ge S, Zhu H, Cao X, Li N. The influence of normal load and sliding speed on frictional properties of skin. Journal of Bionic Engineering 2008:5:33-8

[7] Prevost A, Scheibert J, Debregeas G. Effect of fingerprints orientation on skin vibrations during tactile exploration of textured surfaces. Commununicative \& Integrative Biology 2009 Sep-Oct;2(5):422-4.

[8] Johansson RS, Lofvenberg J. Regional differences and inter-individual variability in sensitivity to vibration in the glabrous skin of the human hand. Brain Research 1984;301:65-72.

[9] Jenmailm P, Birznierks I, Goodwin AW, Johansson RS. Influence of object shape on responses of human tactile afferents under conditions characteristic of manipulation. European Journal of Neuroscience 2003;18:164-76.

[10] Lundstrom R, Johansson RS. Acute impairment of the sensitivity of skin mechanoreceptive units caused by vibration exposure of the hand. Ergonomics 1986;29(5):687-98.

[11] Talbot WH, Darian-Smith I, Kornhuber HH, Mountcastle VB. The sense of flutter-vibration: comparison of the human capacity with response patterns of mechanoreceptive afferents from the monkey hand. Journal of Neurophysiology 1968;31:301-34.

[12] Bolanowski SJ, Gescheider GA, Verrillo RT, Checkosky CM. Four channels mediate the mechanical aspects of touch. Journal of Acoustical Society of America 1988;84:1680-94.

[13] Goodwin AW, John KT, Marceglia AH. Tactile discrimination of curvature by humans using only cutaneous information from the fingerpads. Experimental Brain Research 1991;86:663-72.

[14] Johnson KO. The roles and functions of cutaneous mechanoreceptors. Current Opinion in Neurobiology 2001;11:455-61.

[15] Johnson KO, Yoshioka T, Vega-Bermudez F. Tactile functions of mechanoreceptive afferents innervating the hand. Journal of Clinical Neurophysiology 2000; $17: 539-58$

[16] Goodwin AW, Wheat HE. Magnitude estimation of contact force when objects with different shapes are applied passively to the fingerpad. Somatosensory and Motor Research 1992;9:339-44.

[17] VegA-Bermudez F, Johnson KO. SA I and RA receptive fields, responses variability, and population responses mapped with a probe array. Journal of Neurophysiology 1999;81:2701-10.

[18] Brisben AJ, Hsiao SS, Johnson KO. Detection of vibration transmitted through an object grasped in a hand. Journal of Neurophysiology 1999;81:1548-58.

[19] Bolanowski SJ, Gescheider GA, Verrillo RT. Hairy skin: psychophysical channels and their physiological substrates. Somatosensory and Motor Research 1994;11:279-90.

[20] Olausson H, Wessberg J, Kakuda N. Tactile directional sensibility: peripheral neural mechanisms in man. Brain Research 2000;866:178-87.

[21] Edin BB, Westling G, Johansson RS. Independent control of human fingertip forces at individual digits during precision lifting. Journal of Physiology 1995;487:243-51

[22] Collins DF, Prochazka A. Movement illusions evoked by ensemble cutaneous input from the dorsum of the human hand. Journal of Neurophysiology 1996;496:857-71.

[23] Hollins M, Goble A. Perception of the length of voluntary movements. Somatosensory Research 1988:5:335-48.
[24] Ino S, Shimizu S, Odagawa T, Sato M, Tanahashi M, Izumi T, Ifukube T. A tactile display for presenting quality of materials by changing the temperature of skin surface. IEEE International Workshop on Robot and Human Communication 1993:220-4.

[25] Hollins M, Bensmaia SJ, Risner R, 1998, The duplex theory of tactile texture perception. In: Proceedings of fourteenth annual meeting of the international society for psychophysics, pp. 115-120.

[26] Hollins M, Bensmaia SJ, Karlof K, Young F. Individual differences in perceptua space for tactile textures: evidence from multidimensional scaling. Perception and Psychophysics 2000;62:1534-44.

[27] Hollins M, Risner R. Evidence for the duplex theory of tactile texture perception. Perception and Psychophysics 2000;62(4):695-705.

[28] Hollins M, Bensmaia SJ, Washburn S. Vibrotactile adaptation impairs discrimination of fine, but not coarse, textures. Somatosensory and Motor Research 2001;18:253-62.

[29] Hollins M, Fox A, Bishop C. Imposed vibration influences perceived tactile smoothness. Perception 2000;29:1455-65.

[30] Hollins M, Lorenz F, Harper D. Somatosensory coding of roughness: the effect of texture adaptation in direct and indirect touch. Journal of Neuroscience 2006;26:5582-8.

[31] Gescheider GA, Wright JH. Effects of sensory adaptation on the form of the psychophysical magnitude function for cutaneous vibration. Journal of Experimental Psychology 1968;77:308-13.

[32] Lederman SJ, Taylor MM. Fingertip force, surface geometry, and perception of roughness by active touch. Perception \& Psychophysics 1972;12(5):401-8.

[33] Lederman SJ, Loomis JM, Williams DA. The role of vibration in the tactua perception of roughness. Perception \& Psychophysics 1982;32(2):109-16.

[34] Lederman SJ. Tactual roughness perception: spatial and temporal determinants. Canadian Journal of Psychology 1983;37(4):498-511.

[35] Bensmaia SJ, Hollins M. The vibrations of texture. Journal of Somatosens Mot Res 2003;20(1):33-43.

[36] Scheibert J, Leurent S, Prevost A, Debregeas G, The role of fingerprints in the coding of tactile information probed with a biomimetic sensor. Science, 323 . 1503-1506.

[37] Asserin J, Zahouani H, Humbert Ph, Couturaud V, Mougin D. Measurements of the friction coefficient of the human skin in vivo. Quantification of the cutaneous smoothness. Collids and Surfaces 2000;19:1-12.

[38] Derler A, Schrade U, Gerhardt LC. Tribology of human skin and mechanical skin equivalents in contact with textiles. Journal of Wear 2007;263:1112-6.

[39] Sivamani RK, Goodman J, GITIS N, Maibach HI. Friction coefficient of skin in real-time. Skin Research and Technology 2003:9:235-9.

[40] Sivamani RK, Goodman J, GITIS NV, Maibach HI. Coefficient of friction: tribological studies in man-an overview. Skin Research and Technology 2003;9:227-34

[41] Tang W, Bhushan B. Adhesion, friction and wear characterization of skin and skin cream using atomic force microscope. Colloids and Surfaces B: Biointerfaces 2010;76:1-15.

[42] Fagiani R, Massi F, Chatelet E, Berthier Y, Sestieri A. Experimental analysis of friction induced vibrations at the finger contact surface. Proceedings of the Institution of Mechanical Engineers, Part J: Journal of Engineering Tribology 2009, doi:10.1243/13506501JET722.

[43] Lobontiu N. Compliant mechanisms design of flexure hinges.CRC Press; 2002

[44] Fagiani R, Massi F, Chatelet E, Berthier Y, Dynamic analysis of surface scanning for tactile perception, ESDA2010-ASME 2010 10th biennial conference on engineering systems design and analysis, Istanbul, Turkey (12-14 July 2010).

[45] Phillips JR, Johansson RS, Johnson KO. Responses of human mechanoreceptive afferents to embossed dot arrays scanned across fingerpad skin. Journal of Neuroscience 1992;12(3):827-39.

[46] Adams MJ, Briscoe BJ, Johnson SA. Friction and lubrication of human skin Tribology Letters 2007;26(3):239-53.

[47] Barnes CI, Childs THC, Henson B, Soutee $\mathrm{CH}$. Surface finish and touch-a case study in a new human factors tribology. Journal of Wear 2004:740-50.

[48] Yoshika T, Bensmaia SJ, Craig JC, Hsiao SS. Texture perception through direct and indirect touch: an analysis of perceptual space for tactile textures in two modes of exploration. Journal of Somatosensory and Motor Research 2007; 24(1-2):53-70.

[49] Akay A, Sound and vibration from friction between soft materials and under light loads. In: 36th Leeds-Lyon symposium on tribology, Lyon, 2009.

[50] Martinot F, The influence of surface commensurability on roughness perception with a bare finger. In: Proceedings of eurohaptics 2006, Paris, France 2006. 